\title{
Analysis of voltage stability uncertainty using stochastic response surface method related to wind farm correlation
}

\author{
Zhaoxing $\mathrm{Ma}^{1 *} \mathbb{D}$, Hao Chen $^{2}$ and Yanli Chai ${ }^{3}$
}

\begin{abstract}
Wind speed follows the Weibull probability distribution and wind power can have a significant influence on power system voltage stability. In order to research the influence of wind plant correlation on power system voltage stability, in this paper, the stochastic response surface method (SRSM) is applied to voltage stability analysis to establish the polynomial relationship between the random input and the output response. The Kendall rank correlation coefficient is selected to measure the correlation between wind farms, and the joint probability distribution of wind farms is calculated by Copula function. A dynamic system that includes system node voltages is established. The composite matrix spectral radius of the dynamic system is used as the output of the SRSM, whereas the wind speed is used as the input based on wind farm correlation. The proposed method is compared with the traditional Monte Carlo (MC) method, and the effectiveness and accuracy of the proposed approach is verified using the IEEE 24-bus system and the EPRI 36-bus system. The simulation results also indicate that the consideration of wind farm correlation can more accurately reflect the system stability.
\end{abstract}

Keywords: Power system, SRSM, Correlation, Wind farm

\section{Introduction}

Around the world, power systems have witnessed increased amount of renewable and dispersed generation, especially wind power and solar power. Renewable energy is a useful supplement to traditional energy sources, but is different from the traditional form of energy because of its uncertainty and intermittency. The renewable energy is connected to power grid by concentrated form or distributed form, bringing many uncertainties to power system voltage stability as well as new problems and challenges to researchers. If system voltage stability is evaluated in the most severe working model for studying, the results are often too conservative, and therefore, a new effective way should be investigated. This paper propose a method to investigate the impact of stochastic uncertainty of grid-connected wind power generation on power system voltage stability by structure dynamic systems that include node voltages.

The impact of stochastic power injections on power flows and voltage profile is a widely studied topic since the 1970s

\footnotetext{
* Correspondence: mazhaoxingapple@126.com

${ }^{1}$ College of Automation Engineering, Qingdao University of Technology, Qingdao 266033, China

Full list of author information is available at the end of the article
}

[1]. The probabilistic analysis was firstly introduced into studying power system small signal stability by Burchett and Heydt in [2]. A series of work later on [3-6] have further improved the various aspects of the analytical method of power system probabilistic small signal stability. In $[7,8]$, a method of probabilistic analysis was proposed to directly calculate the probabilistic density function of critical eigenvalues of a large scale power system from the probabilistic density function of gird connected multiple sources of wind power generation to investigate the impact of stochastic uncertainty of grid-connected wind generation on power system small-signal stability [9]. Reference [10] presented a comparative analysis of the performance of three efficient estimation methods when applied to the probabilistic assessment of small-disturbance stability of uncertain power systems. In [11], an analytical approach was proposed to involve the effects of correlation of wind farms in probabilistic analytical multi-state models of wind farms output generation. Reliability models of wind farms considering wind speed correlation are proposed in [12].

In this paper, power system voltage stability is analyzed using the stochastic response surface method (SRSM). The algebraic equations that contain the voltages are 
converted into a differential system with system node voltages. Then, the output of the SRSM is the output of the composite matrix spectral radius of the dynamic system is constructed, and is used to judge the stability of the system voltage. The IEEE 24-node system and the EPRI 36-node system with wind power are considered as two examples to verify the accuracy of the proposed analysis method.

\section{Discussion}

\subsection{System model analysis}

In power system stability analysis, a power system is characterized by the set of nonlinear dynamic equations as:

$$
\begin{aligned}
\dot{x} & =f(x, y, \tau) \\
0 & =g(x, y, \tau)
\end{aligned}
$$

where $f$ and $g$ express the system dynamic equations and the algebraic equations, respectively. $x$ represents the state variables, $y$ represents the algebraic variables of the node voltage magnitudes and angles, and $\tau$ is the control parameters.

Neglecting the resistances, the algebraic equations that consist of the algebraic variables of the node voltage magnitudes and angles can be shown as:

$$
\begin{aligned}
& P_{L i}+\sum_{j=1}^{m+n} B_{i j} V_{i} V_{j} \sin \left(\alpha_{i}-\alpha_{j}\right)=y_{p i}=0 \\
& -Q_{L i}+\sum_{j=1}^{m+n} B_{i j} V_{i} V_{j} \cos \left(\alpha_{i}-\alpha_{j}\right)=y_{Q i}=0
\end{aligned}
$$

$i=n+1, \cdots, n+m$.

where $P_{L i}$ and $Q_{L i}$ are the $i$ th node active and reactive power, respectively. $V_{i}$ and $V_{j}$ are the voltage of the $i$ th node and the $j$ th generator bus, respectively. $B_{i j}$ represents the reactance of the admittance matrix, and $\alpha_{i}$ is the $i$ th bus phase angle. If $1 \leq j \leq n$, then $\alpha_{j}=\delta_{j}$, where $\delta_{j}$ is the generator rotor angle of the $j$ th machine. $n$ is the number of generator buses and $m$ is the number of load buses. $P_{L i}$ and $Q_{L i}$ are functions of $V_{i}$ and $\alpha_{i}$.

Equations (3 and 4) represents a pure dynamic system and taking derivative of $(3,4)$ leads to the dynamic quantity $\dot{V}_{i}$ and $\dot{\alpha}_{i}[12,13]$ as:

$$
{ }^{\partial y_{P i}} /{ }_{\partial t}={ }^{\partial y_{P i}} /{ }_{\partial V} \dot{V}+{ }^{\partial y_{P i}} / \partial \alpha \dot{\alpha}+{ }^{\partial y_{P i}} / \partial \dot{\delta}=0
$$

$$
{ }^{\partial y_{Q i}} /_{\partial t}={ }^{\partial y_{Q i}} /_{\partial V} \dot{V}+{ }^{\partial y_{Q i}} /{ }_{\partial \alpha} \dot{\alpha}+{ }^{\partial y_{Q i}} /{ }_{\partial \delta} \dot{\delta}=0
$$

$$
i=n+1, \cdots, n+m
$$

It is more convenient to represent the generator dynamic equations as follows:

$$
\begin{aligned}
& \dot{\delta}_{i}=\omega_{i} \\
& M_{i} \dot{\omega}_{i}=P_{m i}-\frac{M_{i}}{M_{T}} P_{C O I}-B_{i, i+n} E_{g i} V_{i+n} \sin \left(\delta_{i}-\psi_{i+n}\right)
\end{aligned}
$$

where $\quad \delta_{i}=\bar{\delta}_{i}-\delta_{0}, \quad \omega_{i}=\bar{\omega}_{i}-\omega_{0}, \quad \psi_{i}=\bar{\psi}_{i}-\delta_{0}, \quad M_{T}$ $=\sum_{i=1}^{n} M_{i}, \quad \delta_{0}=\frac{1}{M_{T}} \sum_{i=1}^{n} M_{i} \bar{\delta}_{i}, \quad \omega_{0}=\frac{1}{M_{T}} \sum_{i=1}^{n} M_{i} \bar{\omega}_{i}, \quad P_{C O I}$ $=\sum_{i=1}^{n} P_{m i}-\sum_{i=n+1}^{n+N} P_{L i} . P_{L i}$ is the active load at each node and $P_{m i}$ is the input mechanical power. $\bar{\delta}_{i}$ and $\bar{\omega}_{i}$ are the rotor angle and angular speed of the $i$ th machine, respectively. $\delta_{0}$ and $\omega_{0}$ are the centers of angle and angular speed, respectively. $M_{i}$ and $E_{g i}$ are the $i$ th machine inertia and internal voltage, respectively. $B$ represents the reactance of the admittance matrix. $n$ is the number of generators, $V_{i+n}$ and $\bar{\psi}_{i+n}$ are the generator bus voltage and phase angle, respectively. $N$ is the number of non-generator buses in the power system.

From $(5,6)$, there is

$$
\begin{aligned}
& \partial y_{P i} / \partial V^{\dot{V}+}{ }^{\partial y_{P i}} / \partial \alpha^{\dot{\alpha}=-{ }^{\partial y_{P i}}} / \partial \delta^{\dot{\delta}} \\
& \partial y_{Q i} / \partial V^{\dot{V}+}{ }^{\partial y_{Q i}} /_{\partial \alpha} \dot{\alpha}=-{ }^{\partial y_{Q i}} / \partial \delta^{\dot{\delta}}
\end{aligned}
$$

From $(9,10)$, it yields

$$
\left(\begin{array}{ll}
A(\tau) & B(\tau) \\
C(\tau) & D(\tau)
\end{array}\right)\left(\begin{array}{c}
\dot{V} \\
\dot{\alpha}
\end{array}\right)=-\left(\begin{array}{c}
E(\tau) \dot{\delta} \\
F(\tau) \dot{\delta}
\end{array}\right)
$$

where, $A(\tau)={ }^{\partial y_{P}} / \partial V, B(\tau)=^{\partial y_{P}} / \partial \alpha, C(\tau)={ }^{\partial y_{Q}} / \partial V$, $D(\tau)={ }^{\partial y_{Q}} / \partial \alpha, E(\tau)={ }^{\partial y_{P}} / \partial \delta$ and $F(\tau)={ }^{\partial y_{Q}} / \partial \delta$. Define $\tau=\left(\delta^{\prime}, \omega^{\prime}, V^{\prime}, \alpha^{\prime}\right)$. 
Substituting $(7,8)$ into $(11)$, and solving the dynamic quantity $\dot{V}_{i}$ and $\dot{\alpha}_{i}$ yield:

$$
\left(\begin{array}{c}
\dot{V} \\
\dot{\alpha}
\end{array}\right)=-\left(\begin{array}{ll}
A(\tau) & B(\tau) \\
C(\tau) & D(\tau)
\end{array}\right)^{-1}\left(\begin{array}{l}
E(\tau) \omega \\
F(\tau) \omega
\end{array}\right)
$$

where $V=\left(V_{n+1}, \ldots, V_{n+m}\right)^{\prime}, \alpha=\left(\alpha_{n+1}, \ldots, \alpha_{n+m}\right)^{\prime}, \omega=\left(\omega_{1}\right.$, $\left.\ldots, \omega_{n}\right)^{\prime}, \delta=\left(\delta_{1}, \ldots, \delta_{n}\right)^{\prime}$. The equation can also be expressed as:

$$
\dot{x}=f(x, \omega)
$$

where $x=(V, \alpha)^{\prime}$.

Using (13), a dynamic system can be constructed that contains power system node voltages. A node voltage state equation and its Jacobian matrix can then be established and used to meet the uncertainty of wind power generation. The uncertain elements that are included in the wind power are considered as the input, and a single element that can measure the system voltage stability is considered as the output. After the application of "black box algorithm", it can analyze the influence of the uncertainties on system voltage stability.

Setting $\boldsymbol{J}$ the Jacobian matrix of system (13) at the equilibrium. If the real parts of all the eigenvalues of the Jacobian matrix $\boldsymbol{J}$ is negative, the system (13) is stable; otherwise, the system can become unstable. Thus, a new simple and effective lemma [14] is given as follows:

Lemma 1 If the spectral radius $\rho(J)$ of matrix $\boldsymbol{J}$ satisfies $\rho(\boldsymbol{J})<1$, then the matrix $\boldsymbol{J}$ is a convergence matrix.

Theorem 1 Assuming that $(\boldsymbol{I}-\boldsymbol{J})^{-1}$ exists and $(\boldsymbol{I}+\boldsymbol{J})(\boldsymbol{I}$ $-J)^{-1}$ converges, the real parts of all the eigenvalues of $\boldsymbol{J}$ are negative, where I represents the identity matrix.

Theorem 2 Assuming that $(\boldsymbol{I}-\boldsymbol{J})^{-1}$ exists and $(\boldsymbol{I}+\boldsymbol{J})(\boldsymbol{I}$ $-J)^{-1}$ dose not converge, the real parts of all the eigenvalues of $\boldsymbol{J}$ are nonnegative.

A power system with wind plant is considered as an example for further elaboration. The wind speed is set to $v_{w}$, which is considered as the uncertain input element. $\boldsymbol{A}$ is the system Jacobi matrix at the equilibrium point, so the complex matrix $\left((\boldsymbol{I}+\boldsymbol{A})(\boldsymbol{I}-\boldsymbol{A})^{-1}\right)$ can be founded. The spectral matrix of the complex matrix that can be used to judge the stability of the system voltage can be taken as the output. After calculation using the black box algorithm, as the spectral radius $\rho\left((\boldsymbol{I}+\boldsymbol{J})(\boldsymbol{I}-\boldsymbol{J})^{-1}\right)$ of the matrix $(\boldsymbol{I}+\boldsymbol{J})(\boldsymbol{I}-\boldsymbol{J})^{-1}$ satisfies $\rho\left((\boldsymbol{I}+\boldsymbol{J})(\boldsymbol{I}-\boldsymbol{J})^{-1}\right)<1$, all of the real parts of the eigenvalues of matrix $\boldsymbol{J}$ are negative and the existing dynamic system voltage is stable. In contrast, if the spectral radius $\rho\left((\boldsymbol{I}+\boldsymbol{J})(\boldsymbol{I}-\boldsymbol{J})^{-1}\right)$ satisfies $\rho\left((\boldsymbol{I}+\boldsymbol{J})(\boldsymbol{I}-\boldsymbol{J})^{-1}\right) \geq 1$, the system voltage is unstable.

Thus, the relationship between the elements of wind power uncertainty and the system voltage stability is established, and the system voltage stability state can be assessed.

\subsection{Stochastic response surface method analysis}

SRSM improves the computational efficiency and accuracy of probability analysis through special reconnaissance and polynomial chaos expansion model output, which is considered to be deterministic classical response surface method [15]. SRSM significantly improves the efficiency and reliability of the analysis, and avoids the iterative computation of traditional methods [16-18].

The principle of SRSM is based on the probability distribution of known parameters, and the response approximation expressed as a polynomial function of the model parameters. Set the response $Y$ as a function of the uncertain parameter $x$, which represents the form of the model as:

$$
Y=u(x)
$$

The estimated value $\hat{Y}$ can correctly describe the characteristics of the response $Y$ and is expressed as:

$$
\hat{Y}=\hat{u}(x)
$$

where $\hat{u}(x)$ is polynomial function.

The estimated value $\hat{Y}$ of the response is constructed by SRSM with Hermite polynomial, and $p$-rank expression as:

$$
H_{p}(x)=(-1)^{p} e^{\frac{x^{2}}{2}} \frac{d^{p}}{d x^{p}}\left(e^{-\frac{x^{2}}{2}}\right)
$$

It satisfies the orthogonality:

$$
\int_{-\infty}^{+\infty} e^{-\frac{x^{2}}{2}} H_{p}(x) H_{q}(x)=0
$$

SRSM is able to make the output model as a polynomial chaos expansion model with the standard normal distribution random variables:

$$
\begin{gathered}
y=a_{0}+\sum_{i 1=1}^{n} a_{i 1} \eta_{1}\left(\phi_{i 1}\right)+\sum_{i 1=1 i 2=1}^{n} \sum_{i 12}^{i 1} a_{i 12} \eta_{2}\left(\phi_{i 1}, \phi_{i 2}\right) \\
+\sum_{i 1=1 i 2=1 i 3=1}^{n} \sum_{i 1}^{i 2} a_{i 12 i 3} \eta_{3}\left(\phi_{i 1}, \phi_{i 2}, \phi_{i 3}\right)+\cdots
\end{gathered}
$$

where $a_{0}, a_{i 1}$ and $a_{i 1 i 2}$ are the determining factors that need be determined; $n$ is number of input random variables; $\phi_{i j}$ is the $j$ th standard normal distribution of random variables. $\eta_{p}\left(\phi_{i 1}, \phi_{i 2}, \ldots, \phi_{i p}\right)$ is a $p$-rank Hermite orthogonal polynomial; and can be shown as:

$$
\eta_{p}\left(\phi_{i 1}, \cdots, \phi_{i p}\right)=(-1)^{p} e^{\frac{1}{2} \psi^{\prime} \psi} \frac{\partial^{p}}{\partial \phi_{i 1} \cdots \partial \phi_{i p}} e^{-\frac{1}{2} \psi^{\prime} \psi}
$$

where $\Psi$ represents the vector of $\left\{\phi_{i k}\right\}_{k=1}^{p}, p \geq 1$. Solving $\eta_{p}\left(\phi_{i 1}, \phi_{i 2}, \ldots, \phi_{i p}\right)$, and substituting into the (14) can obtain the expression of the output model $y$. 


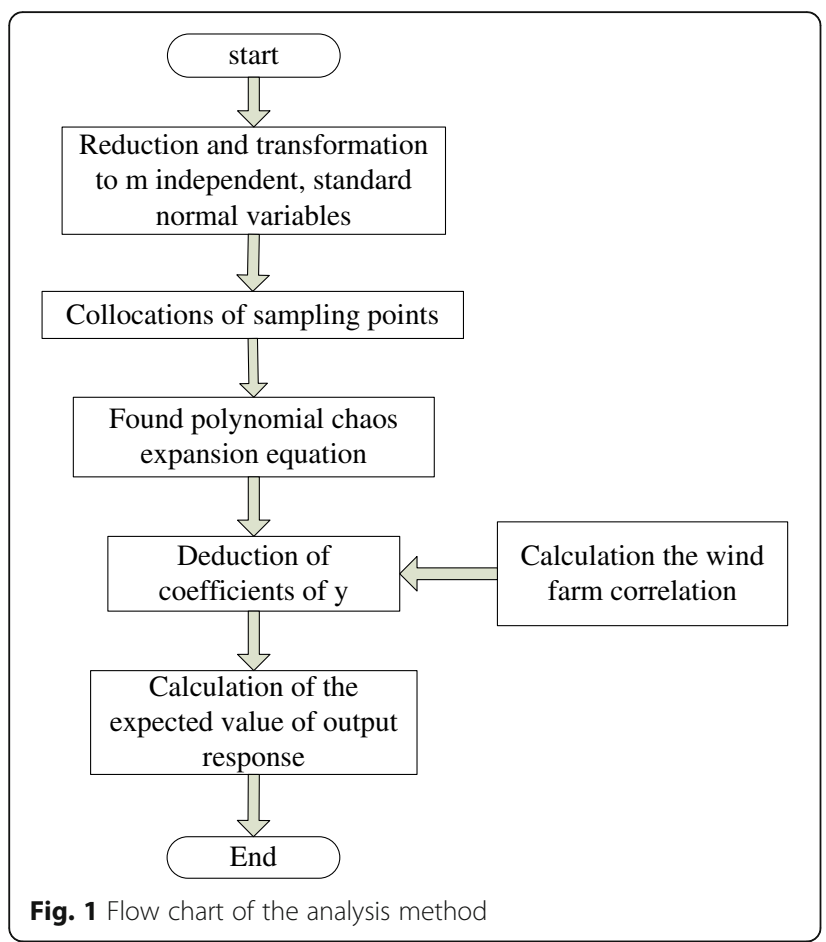

In the following, a 2-rank expansion with $n$ random variables is taken as an example for illustration. The 2-rank expansion is given as:

$$
\begin{aligned}
y_{2}\left(\phi_{1}, \phi_{2}\right) & =a_{0}+a_{1} \phi_{1}+a_{2} \phi_{2}+a_{3}\left(\phi_{1}^{2}-1\right) \\
& +a_{4}\left(\phi_{2}^{2}-1\right)+a_{5} \phi_{1} \phi_{2}
\end{aligned}
$$

For (16), six certainty coefficients need be solved in 2-rank output model $y_{2}$. Thus, the number of the

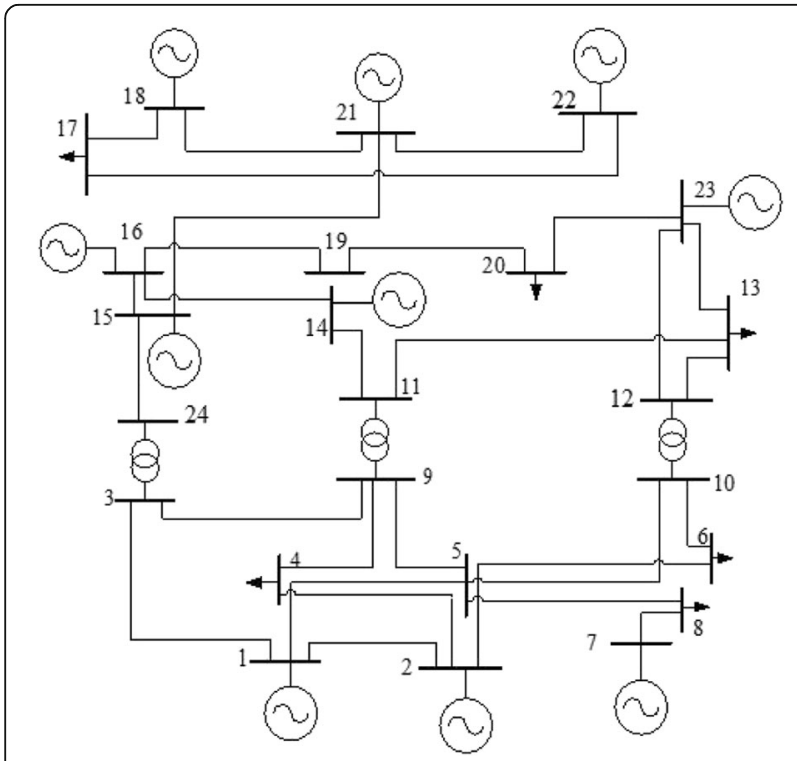

Fig. 2 The IEEE 24-bus system
Table 1 Parameters of wind farms

\begin{tabular}{lll}
\hline Wind farms & 1 & 2 \\
Fans & 40 & 20 \\
Rated capacity $(\mathrm{MW})$ & 0.6 & 1.5 \\
Cut in wind speed $(\mathrm{m} / \mathrm{s})$ & 4 & 3 \\
Cut out wind speed $(\mathrm{m} / \mathrm{s})$ & 22 & 24 \\
Rated wind speed $(\mathrm{m} / \mathrm{s})$ & 14 & 15 \\
\hline
\end{tabular}

certainty coefficients $a_{i}$ be solved in the 2-rank model is $1+2 n+1 / 2(n(n-1))$ with $n$ random variables.

In applying SRSM, the most important task is to solve the unknown coefficient $a_{i}$. Both the probability distribution method and the efficient regression method can be used to solve the unknown coefficient, although, the efficiency of the efficient regression method is better and the results are more robust. As the input variable quantities are $\phi_{1 i}$ and $\phi_{2 i},(16)$ can be represented as:

$$
\begin{aligned}
y_{2 i}\left(\phi_{1 i}, \phi_{2 i}\right) & =a_{0}+a_{1} \phi_{1 i}+a_{2} \phi_{2 i}+a_{3}\left(\phi_{1 i}^{2}-1\right) \\
& +a_{4}\left(\phi_{2 i}^{2}-1\right)+a_{5} \phi_{1 i} \phi_{2 i}
\end{aligned}
$$

To calculate the unknown coefficients $a_{i}$, some sample points with forms as $\left(\phi_{1 m}, \phi_{2 m}\right)$ are required to be selected. In this paper, $\phi$ describes the wind speed following probability distribution, and y expresses the degree of voltage instability by (16) and (17). Thus, the relation that is related to power system is established for analysis.

Equation (17) is the 2-rank expansion model, and the roots $(0, \sqrt{3},-\sqrt{3}$ of the 3-rank Hermite polynomials can be selected with a total of nine sample points. If the number of random variables is more than 3 , the number of sample points is two times larger than that of the unknown factor, and thus large amount of calculation is required. However, the selected sample points are in the standard normal distribution space, and therefore, it is necessary to convert them to the original space. The transformation of the original space sample points corresponds to the real response value, and the unknown coefficients $a_{i}$ can be obtained using the least square method for solving linear equations.

\subsection{Copula theory correlation analysis} 2.3.1 Copula function definition

Assuming $H(\cdot, \cdot)$ is the joint distribution function of $F(\cdot)$ and $G(\cdot)$ with marginal distribution, there exists a Copula function $C(\cdot, \cdot)$ satisfying:

$$
H(x, y)=C(F(x), G(y))
$$

The density function of the distribution function $H(\cdot, \cdot)$ can be derived by the density function $C(, \cdot)$ of the 


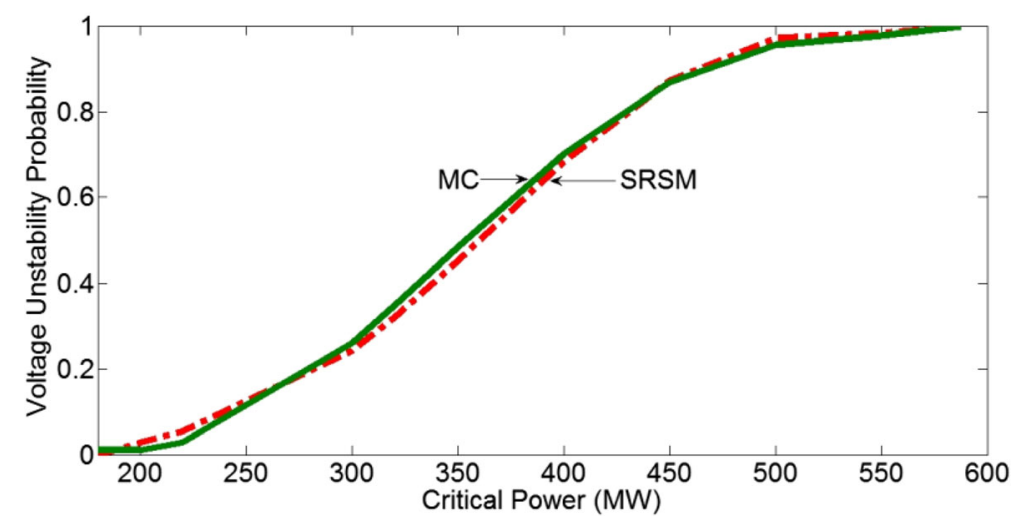

Fig. 3 Cumulative density distribution of voltage instability with critical power

Copula function and the edge distribution function $F(\cdot)$ and $G(\cdot)$ as:

$$
h(x, y)=c(F(x), G(y)) f(x) g(y)
$$

where $c(u, v)={ }^{\partial C(u, v)} / \partial u \partial v, u=F(x), v=G(y) ; f(\cdot)$ and $g(\cdot)$ are the density functions of $F(\cdot)$ and $G(\cdot)$, respectively. In this paper, wind power output sequences of the two wind power plants are $x$ and $y$, and their distribution functions are $F(x)$ and $G(y)$, respectively. $u=F(x), v=$ $G(y)$. H is the copula function of $F(x)$ and $G(y)$.

In this paper, Frank Copula function [19-22] is considered as the connection function of joint probability distribution of wind farms. The respective distribution function and density function of Frank Copula can be expressed as:

$$
C_{F}(u, v ; \beta)=-\frac{1}{\beta} \ln \left[1+\frac{\left(e^{-\beta u}-1\right)\left(e^{-\beta v}-1\right)}{e^{-\beta}-1}\right]
$$

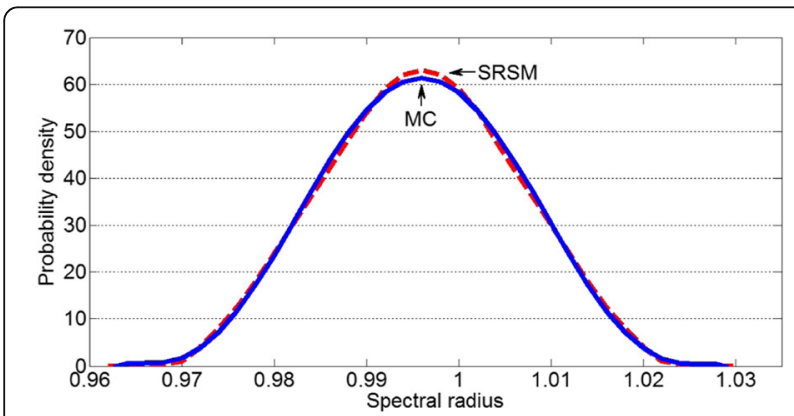

Fig. 4 Probability density of spectral radius with $380 \mathrm{MW}$ wind power generation

$$
c_{F}(u, v ; \beta)=\frac{-\beta\left(e^{-\beta}-1\right) e^{-\beta(u+v)}}{\left[\left(e^{-\beta}-1\right)+\left(e^{-\beta u}-1\right)\left(e^{-\beta v}-1\right)\right]^{2}}
$$

where $\beta$ is the relative parameter and $\beta \neq 0$. If $\beta>0$, random variables $u$ and $v$ have positive correlation. If $\beta \rightarrow 0$, random variables $u$ and $v$ tend to be independent. $\beta<0$ show that random variable $u$ and $v$ have negative correlation.

\subsubsection{Correlation analysis}

Since the traditional Pearson's linear correlation coefficient cannot depict the complicated correlation relationship of different wind speed time series, in this paper, Kendall rank correlation coefficient is selected to measure the correlation of wind farm power. Kendall rank correlation coefficient indicates the difference between the probabilities of agreement and inconsistency from randomly selected observations in the samples. Thus, the general form of Kendall rank correlation coefficient can be obtained. Assuming $\left(x_{i}, y_{i}\right)$ and $\left(x_{j}, y_{j}\right)$ are arbitrary 2 possible values of random vector $(\mathrm{X}, \mathrm{Y}),\left(x_{i}, y_{i}\right)$ and $\left(x_{j}, y_{j}\right)$ are independent and identically distributed. Define:

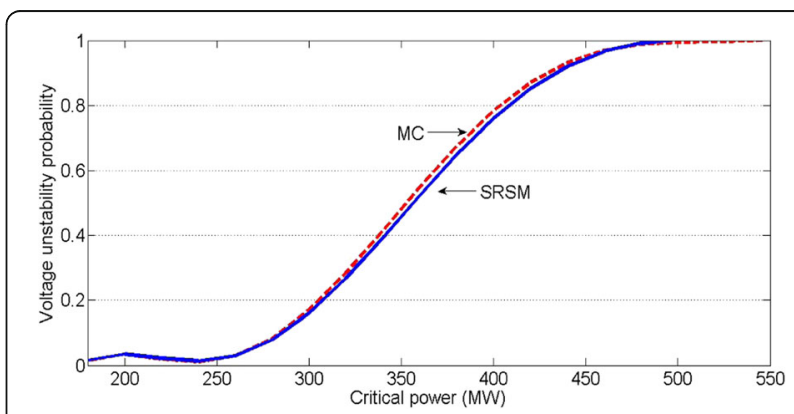

Fig. 5 Cumulative density distribution of voltage instability for correlation $\mathrm{K}=0.162$ 


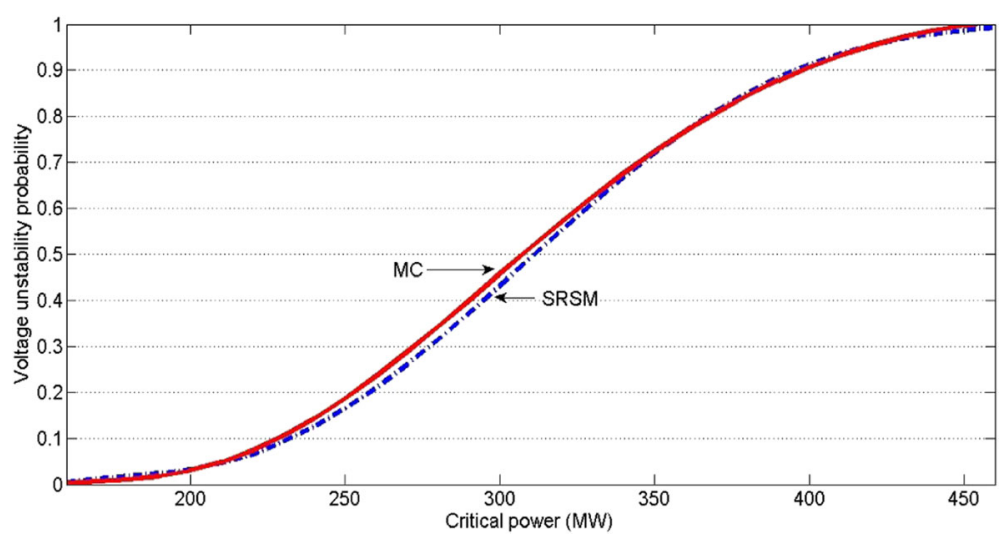

Fig. 6 Cumulative density distribution of voltage instability for correlation $\mathrm{k}=0.257$

$$
\begin{gathered}
\kappa=P\left\{\left(x_{i}-x_{j}\right)\left(y_{i}-y_{j}\right)>0\right\} \\
-P\left\{\left(x_{i}-x_{j}\right)\left(y_{i}-y_{j}\right)<0\right\}
\end{gathered}
$$

as the Kendall rank correlation coefficient, and $\kappa \in[-1,1]$, $i \neq j$. $P$ indicates probability of occurrence. If $\kappa>0$, random variables $X$ and $Y$ have positive correlation; if $\kappa<0$, the random variables have negative correlation. If $\kappa=0$, the correlation between random variables $X$ and $Y$ cannot be determined.

Random variable $P_{1}$ and $P_{2}$ is defined as the output rates of the two wind farms, respectively. $\left(p_{11}, p_{12}, \ldots\right.$, $\left.p_{1 n}\right)$ and $\left(p_{21}, p_{22}, \ldots, p_{2 n}\right)$ are the respective sample space of random variable $P_{1}$ and $P_{2}, n$ is the sample size, which establishes a one-to-one relationship with $p_{1 i}$ and $p_{2 i}$.

The relation between the Kendall rank related coefficient $\kappa$ and the related parameters $\beta$ of Frank Copula function can be expressed as:

$$
\kappa=1+\frac{4}{\beta}\left[D_{k}(\beta)-1\right]
$$

where $D_{k}(\beta)=\frac{k}{\beta^{k}} \int_{0}^{\beta} \frac{t^{k}}{e^{t}-1} d t, k=1$.

\subsection{Wind power uncertainty analysis}

The relationships between active power $P_{e}$ that is supplied by the wind generation source and wind speed $v$ are expressed as $[14,20]$ :

Table 2 Average error indices of IEEE 24-node system in different correlation

\begin{tabular}{lc}
\hline Correlation coefficient & Average error \\
\hline 0.162 & 0.039 \\
0.257 & 0.041 \\
\hline
\end{tabular}

$$
P_{e}=\left\{\begin{array}{cc}
c+d \nu, & \text { as } v_{\text {in }} \leq \nu \leq v_{r} \\
P_{0}, & \text { as } v_{r} \leq \nu \leq v_{\text {out }} \\
0, & \text { others }
\end{array}\right.
$$

where $v_{\text {in }}$ and $v_{\text {out }}$ are respective cut-in wind speed and cut-out wind speeds, $v_{r}$ is the rated wind speed, $P_{e}$ is the active power generated by the wind farm, and $P_{O}$ is the rated active power. $c$ and $d$ are constants.

In this paper, the wind speed is assumed to follows a Weibull distribution and is shown by variation $\eta$ of standard normal distribution as:

$$
v(t)=\left(-\ln \left[\frac{1}{2}+\left[\frac{1}{2} g_{\operatorname{erg}}\left(\frac{\eta}{\sqrt{2}}\right)\right]\right]\right)^{\frac{1}{t}}
$$

where $g_{\text {erg }}$ is the Gaussian error function.

The analysis process with SRSM is expressed in the next section.

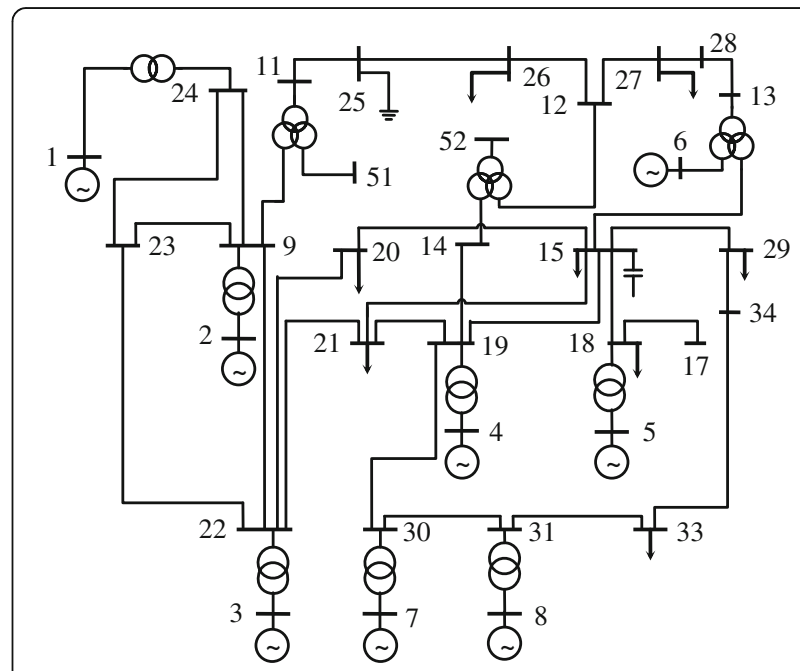

Fig. 7 The single-line diagram of the 36 -node system 


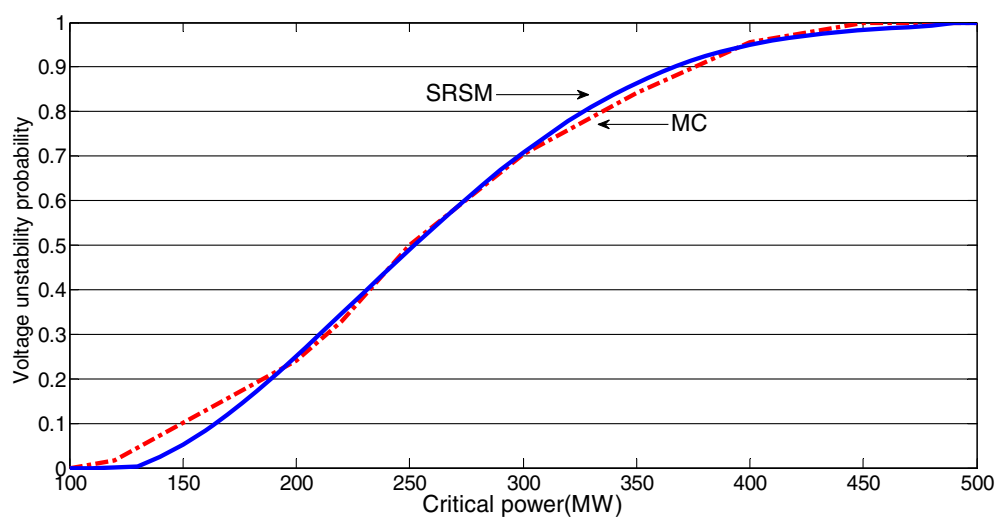

Fig. 8 Cumulative density distribution of voltage instability for correlation $\mathrm{k}=0.280$

\section{Method}

\subsection{Voltage uncertainty analysis with SRSM}

As for actual systems that contains wind power, whose wind speed follows Weibull distribution rather than the normal distribution, it should convert the wind speed as the standard normal distribution to analyze the impact of wind power uncertainty on voltage stability based on SRSM. Some researchers also apply SRSM to analyze uncertainty of power system dynamic simulation [23]. The flow chat for the calculation and analysis of the uncertainty of wind power on stability using SRSM is shown in Fig. 1.

\section{Results}

\subsection{Case studies}

In the test cases, dispersed wind generation is considered in the IEEE 24-bus system shown in Fig. 2 and two wind farms are added into the system at node 1 and 7 , respectively. In all cases presented below, comparisons are made to the Monte Carlo (MC) numerical approach.

\subsection{Case 1}

In this test case, the outputs of the two wind farms are independent from each other. In this paper, MC simulated 6000 times to verify the accuracy and efficiency of SRSM.

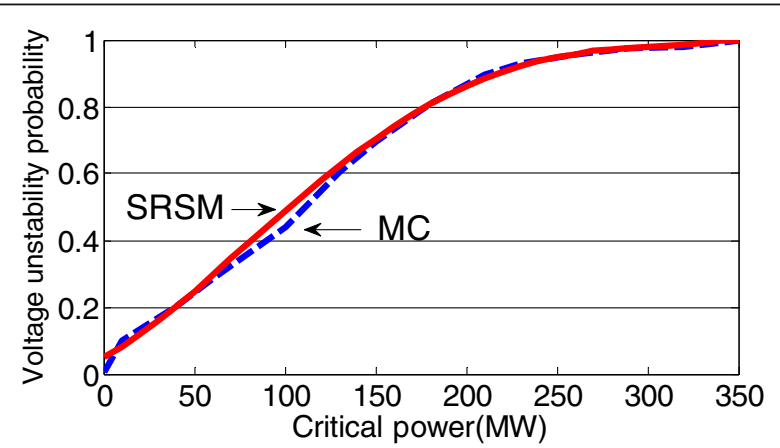

Fig. 9 Cumulative density distribution of voltage instability for correlation $\mathrm{k}=0.794$
To enable a balanced comparison of the accuracy between SRSM and MC, the same number of uncertainties are used for each moment model. The parameters of the two wind farms are shown in Table 1 . In study, the mode of load change is that increase the whole network load simultaneously, with the load of each node increased by the same proportion. In the load direction, the load component of the partial load follows normal distribution.

Figure 3 shows the cumulative probability curve obtained by SRSM and MC method for the IEEE 24-node system, and the voltage instability probability of the system are determined under different load levels.

In Fig. 4, the probability density of spectral radius with $380 \mathrm{MW}$ wind power generation is given. The calculation results also show that SRSM has high accuracy than the MC method, and it can meet the needs for the practical applications.

\subsection{Case 2}

In this test case, two wind farms are correlated each other. Figures 5 and 6 compare the cumulative probability curve obtained by SRSM and MC for the IEEE 24-node system, and the system voltage instability probability are determined under different load levels.

In Figs. 5 and 6, MC was used to verify the accuracy of the proposed SRSM method in the paper, and is can be seen that SRSM method has good accuracy. Under the condition of different correlation coefficients, the average error of voltage instability analysis with SRSM is given in Table 2. Calculation results shown that Kendall rank correlation coefficient method in analyzing voltage instability has good accuracy, and can satisfy the engineering requirement.

The results of numerical calculation show that wind farm correlation had a significant influence on system voltage stability. According to Figs. 5 and 6, the greater the correlation between the wind farms has, the more noticeable influence on system voltage stability. The 
simulation results of case 1 and case 2 shown that the system voltage may reached an instability state is underestimated without considering the correlation, that is underestimate the potential risks. From the simulation results, it also got that the proposed method can reflect accurately the system voltage stability as analyze the voltage stability uncertainty problems.

\subsection{Case 3}

In the test case, dispersed wind generation is considered in the EPRI 36-node system shown in Fig. 7. Two correlated wind farms whit the same parameters as shown in Table 1 are added to the system and are connected at node 4 and 5 , respectively.

In this case, $\mathrm{MC}$ is simulated 4000 times to verify the accuracy and efficiency of SRSM. The system reference power is $100 \mathrm{MW}$ and the 2-rank SRSM polynomial is used for calculation. In the simulation, the load of each node is again increased by the same proportion.

For correlation coefficient $\mathrm{K}=0.280$ and $\mathrm{\kappa}=0.794$, the cumulative density distributions of voltage instability are expressed in Figs. 8 and 9, respectively.

As can be seen from the Figs. 8 and 9, the larger the correlation between the wind farms has, the lower the voltage instability critical power is, and the probability of instability is greater under the same power condition. Under the condition of strong correlation, it is also indicated that more attention should be paid to the voltage instability problem.

\section{Conclusions}

This paper presents a method that establishes a dynamic system including node voltage to study power system voltages stability incorporating wind farm uncertainty. Rather than the eigenvalues of the Jacobi matrix, the criterion of power system voltage stability is given by the spectral radius of the composite matrix. In the study process, the correlation of wind farms is considered, such that the uncertainty of the wind farms and the analysis method are closer to actual systems. The proposed method which uses SRSM to study the uncertainty can provide power system operators with useful real-time estimation of the power system voltage stability with wind power integration. Compared to the traditional methods, e.g. the Monte Carlo method, the proposed one is more efficient.

The analysis and the simulation results also shown that the proposed method has a higher accuracy and has a good application prospect to actual system operation and stability analysis. The effect of the correlation between multiple distributed energy source on system voltage stability will be considered in future research.

\section{Acknowledgements}

This work is supported by project of the Jiangsu Province University Natural Science Research Foundation (14KJB470003).

\section{Authors' contributions}

ZM contributed to the study design and analysis and drafted the manuscript; $\mathrm{HC}$ worked on aspects of the study relating to wind farm correlation; YC was involved in data acquisition and revision of the manuscript. All authors have read and approved the final manuscript.

\section{Competing interests}

The authors declare that they have no competing interests.

\section{Author details}

${ }^{1}$ College of Automation Engineering, Qingdao University of Technology, Qingdao 266033, China. 'Jiangsu Electric Power Company, Nanjing 210024, China. ${ }^{3}$ Jiangsu Normal University, Xuzhou, China.

Received: 6 January 2017 Accepted: 9 May 2017

Published online: 22 May 2017

\section{References}

1. Schilling, M. T., Leite da silva, A. M., Billinton, R., \& Ei-kady, M. A. (1990). Bibliography on power system probabilistic analysis (1962-1988). IEEE Transactions on Power Systems, 5(1), 1-11.

2. Burchett, C., \& Heydt, G. T. (1978). Probabilistic methods for power system dynamic stability studies. IEEE Transactions on Power Systems, 97(3), 695-702.

3. Kim, Y. K., Lee, G. H., Hong, J. P., Hur, J., Lee, B. K., \& Kang, G. H. (2004). Prediction of torque characteristic on barrier-type SRM using stochastic response surface methodology combined with moving least square. IEEE Transactions on Magnetics, 40(2), 738-741.

4. Haesen, E., Bastiaensen, C., Driesen, J., \& Belmans, R. (2009). A probabilistic formulation of load margins in power systems with stochastic generation. IEEE Transactions on Power Systems, 24(2), 951-958.

5. Bai, F. F., Liu, Y., Liu, Y. L., Sun, K., Bhatt, N., et al. (2016). A measurementbased approach for power system instability early warning. Protection and Control of Modern Power System, 1, 1-9.

6. Ai, Q., Fan, S. L., \& Piao, L. J. (2016). Optimal scheduling strategy for virtual power plants based on credibility theory. Protection and Control of Modern Power System, 1, 20-27.

7. Faried, S. O., Billinton, R., \& Aboreshaid, S. (2010). Probabilistic evaluation of transient stability of a power system incorporating wind farms. IET Renewable Power Generation, 4(4), 299-307.

8. Bu, S. Q., Du, W., Wang, H. F., Chen, Z., Xiao, L. Y., \& Li, H. F. (2012). Probabilistic analysis of small-signal stability of large-scale power system as affected by penetration of wind generation. IEEE Transactions on Power Systems, 27(2), 762-770.

9. Wang, C., Shi, L. B., Yao, L. Z., Wang, L. M., Ni, Y. X., \& Bazargan, M. (2010). Modelling analysis in power system small signal stability considering uncertainty of wind generation. IEEE PES SM Paper, 22(5), 1-7.

10. Preece, R., Huang, K. J., \& Milanovie, J. V. (2014). Probabilistic smalldisturbance stability assessment of uncertain power system using efficient estimation methods. IEEE Transactions on Power Systems, 29(5), 2509-2517.

11. Riahinia, S., Abbaspour, A., Fotuhi-Firuzabad, M., \& Moeini-Aghtaie, M. (2015). Impact of correlation on reserve requirements of high windpenetrated power systems. International Journal of Electrical Power\& Energy Systems, 73, 576-583.

12. Chen, F., Li, F. X., Wei, Z. N., Sun, G. Q., \& Li, J. (2015). Reliability models of wind farms considering wind speed correlation and WTG outage. Electric Power Systems Research, 119, 385-392.

13. Mehraeen, S., Jagannathan, S., \& Mariesa, L. C. (2010). Novel dynamic representation and control of power systems with FACTS devices. IEEE Transactions on Power Systems, 25(3), 1542-1554.

14. Ma, Z. X., Gu, W., \& Wan, Q. L. (2014). Study on power system smalldisturbance uncertainty stability considering wind power. IEEJ Transactions on Electrical and Electronic Engineering, 9, 123-128.

15. Bao, H. B., \& Wei, H. (2012). A stochastic response surface method for probabilistic evaluation of the voltage stability considering wind power. Proceedings of the CSEE, 32(13), 77-85.

16. Ren, Z. Y., Li, W. Y., Billinton, R., \& Yan, W. (2015). Probabilistic power flow analysis based on the stochastic response surface method. IEEE Transactions on Power Systems, 26(3), 1-9.

17. Han, D., He, R. M., Ma, J., \& Huang, X. J. (2008). Quantitative uncertainty analysis for power system dynamic simulation based on stochastic response surface method. Automation of Electric Power Systems, 32(20), 11-15. 
18. Han, D., Ma, J., He, R. M., et al. (2010). Dynamic consistency test for power system time-domain simulation. Automation of Electric Power Systems, 34(16), 29-33.

19. Cai, D. F., Shi, D. Y., \& Chen, J. F. (2013). Probabilistic load flow considering correlation between input random variables based on Copula theory. Power System Protection and Control, 41(20), 13-17.

20. Feng, L., Zhang, J. N., Li, G. J., \& Zhang, B. L. (2016). Cost reduction of a hybrid energy storage system considering correlation between wind and PV power. Protection and Control of Modern Power System, 1, 11-19.

21. Gao, Y., \& Billinton, R. (2009). Adequacy assessment of generating systems containing wind power considering wind speed correlation. IET Renewable Power Generation, 3(2), 217-226.

22. Chen, H. (2016). Load forecasting based on modern time series analysis method (pp. 187-208). China Electric Power Press.

23. Zhang, L., Huang, J. D. \& Wang, L. L. (2014). Impact of wind speed correlation on transient stability of power system. Power System Protection and Control, $42(6), 77-81$

\section{Submit your manuscript to a SpringerOpen ${ }^{\circ}$ journal and benefit from:}

- Convenient online submission

- Rigorous peer review

- Open access: articles freely available online

- High visibility within the field

- Retaining the copyright to your article

Submit your next manuscript at $\boldsymbol{\sim}$ springeropen.com 